\title{
Eupatilin treatment inhibits transforming growth factor beta-induced endometrial fibrosis in vitro
}

\author{
Chang-Jin Lee ${ }^{1, *}$, Seon-Hwa Hong,* , Min-Ji Yoon', Kyung-Ah Lee', Dong Hee Choi ${ }^{2}$, Hwang Kwon'2, Jung-Jae Ko', \\ Hwa Seon Koo ${ }^{2}$, Youn-Jung Kang ${ }^{1,2,3}$
}

'Department of Biomedical Science, School of Life Science, ${ }^{2} \mathrm{CHA}$ Fertility Center Bundang, ${ }^{3}$ Department of Biochemistry, School of Medicine, CHA

University, Seongnam, Korea

\begin{abstract}
Objective: Endometrial fibrosis, the primary pathological feature of intrauterine adhesion, may lead to disruption of endometrial tissue structure, menstrual abnormalities, infertility, and recurrent pregnancy loss. At present, no ideal therapeutic strategy exists for this fibrotic disease. Eupatilin, a major pharmacologically active flavone from Artemisia, has been previously reported to act as a potent inducer of dedifferentiation of fibrotic tissue in the liver and lung. However, the effects of eupatilin on endometrial fibrosis have not yet been investigated. In this study, we present the first report on the impact of eupatilin treatment on transforming growth factor beta (TGF- $\beta$ )-induced endometrial fibrosis.

Methods: The efficacy of eupatilin on TGF- $\beta$-induced endometrial fibrosis was assessed by examining changes in morphology and the expression levels of fibrosis markers using immunofluorescence staining and quantitative real-time reverse-transcription polymerase chain reaction. Results: Eupatilin treatment significantly reduced the fibrotic activity of TGF- $\beta$-induced endometrial fibrosis in Ishikawa cells, which displayed more circular shapes and formed more colonies. Additionally, the effects of eupatilin on fibrotic markers including alpha-smooth muscle actin, hypoxia-inducible factor 1 alpha, collagen type I alpha 1 chain, and matrix metalloproteinase-2, were evaluated in TGF- $\beta$-induced endometrial fibrosis. The expression of these markers was highly upregulated by TGF- $\beta$ pretreatment and recovered to the levels of control cells in response to eupatilin treatment.

Conclusion: Our findings suggest that suppression of TGF- $\beta$-induced signaling by eupatilin might be an effective therapeutic strategy for the treatment of endometrial fibrosis.
\end{abstract}

Keywords: Endometrial fibrosis; Eupatilin; Transforming growth factor beta

Received: December 11, 2019 · Revised: December 30, 2019.

Accepted: December 30, 2019

Corresponding author:Youn-Jung Kang

Department of Biochemistry, School of Medicine, CHA University,

43 Beolmal-ro 30beon-gil, Bundang-gu, Seongnam 13503, Korea

Tel: +82-31-881-7174 Fax:+82-31-881-7069 E-mail:yjkang@cha.ac.kr

Co-Corresponding author: Hwa Seon Koo

CHA Fertility Center Bundang, School of Medicine, CHA University,

43 Beolmalro 30beon-gil, Bundang-gu, Seongnam 13503, Korea

Tel:+82-31-780-8701 Fax:+82-31-881-7069 E-mail:hwas0605@cha.ac.kr

Co-Corresponding author: Jung-Jae Ko

Department of Biomedical Science, School of Life Science, CHA University, 43 Beolmal-ro 30beon-gil, Bundang-gu, Seongnam 13503, Korea

Tel:+82-31-881-7133 Fax:+82-31-881-7069 E-mail:highko@chamc.co.kr

*These authors contributed equally to this work.

**This work was supported by funding from a National Research Foundation of Korea (NRF) grant funded by the Korean government (No. 2018R1C1B6003) to YJK.

This is an Open Access article distributed under the terms of the Creative Commons Attribution Non-Commercial License (http://creativecommons.org/licenses/by-nc/4.0/) which permits unrestricted non-commercial use, distribution, and reproduction in any medium, provided the original work is properly cited.

\section{Introduction}

Endometrial fibrosis, the primary pathological feature of intrauterine adhesion, is commonly accompanied by increased deposition and reorganization of extracellular matrix (ECM) proteins, leading to disruptions of endometrial tissue structure, menstrual abnormalities, infertility, and recurrent pregnancy loss [1-5]. Fibrotic progression in the endometrium can be triggered by various factors, including persistent bacterial or viral infections, genetic disorders, or traumatic damage from injury or chemical exposure [6]. Despite the diversity of the causes of endometrial fibrosis, hysteroscopy of fibrotic lesions is only adopted as a current standard surgical resolution for the diagnosis and treatment of this disease [7]. This pathological condition is mostly driven by the interactions of inflammatory cytokines, including interleukin-6, tumor necrosis factor, and transforming growth 
factor beta (TGF- $\beta$ ), the expression of which is observed in infiltrating inflammatory cells, which tend to migrate towards damaged sites of the endometrium $[2,8,9]$. However, the specific cellular and molecular mechanisms underlying endometrial fibrosis have yet to be fully elucidated. TGF- $\beta$ is known to be a key modulator that facilitates fibrotic progression and the induction of cellular trans-differentiation into myofibroblasts with aberrantly increased ECM deposition [10]. It has been previously reported that TGF- $\beta$ is highly expressed in fibrotic tissue in the lung and skin, and conversely, depletion of TGF- $\beta$ was found to attenuate experimentally-induced fibrosis progression in the skin, lung, liver and endometrium [11-14]. Binding of TGF- $\beta$ to its specific receptor triggers intracellular signal transmission via Smad-dependent and Smad-independent pathways to induce fibrotic progression. The TGF- $\beta$-induced expression of alpha-smooth muscle actin ( $\alpha-S M A)$ requires Smad3 phosphorylation, which facilitates a remarkable increase in collagen deposition. This process is accompanied by upregulation of the expression of tissue inhibitors of metalloproteinases, which inhibit the activity of ECM-degradative enzymes [15-17]. However, attempts to block the transmission of TGF- $\beta$ signaling pathway as candidate anti-fibrotic therapeutic strategies still have limitations due to the characteristics of TGF- $\beta$ as a multifunctional physiological mediator. Eupatilin (5,7-dihydroxy-3', 4',6-trimethoxyflavone), which is a major pharmacologically active flavone that has been isolated from Artemisia species, has been reported to suppress TGF- $\beta$ receptor signaling by interfering with actin polymerization and acting as a potent inducer of dedifferentiation to reduce fibrotic activity in the liver and lung $[18,19]$. However, the effects of eupatilin in endometrial fibrosis have not yet been assessed. Since a critical challenge in endometrial fibrotic disease is to understand the progression and prevention of this complex disease entity, it is crucial to identify the cellular and molecular mechanisms underlying endometrial fibrosis. In this study, we present the first report on the impact of eupatilin treatment on TGF- $\beta$-induced endometrial fibrosis.

\section{Methods}

\section{Cell culture and reagents}

Ishikawa cells (a well-differentiated human endometrial adenocarcinoma cell line), obtained from American Type Cell Culture (Manassas, VA, USA), were maintained in Dulbecco's Modified Eagle Medium/F12 medium (Gibco, Grand Island, NY, USA) supplemented with $10 \%$ fetal bovine serum (Gibco), $100 \mathrm{U} / \mathrm{mL}$ penicillin (Gibco), $100 \mathrm{mg} / \mathrm{mL}$ streptomycin (Gibco) and $2 \mathrm{mM}$ L-glutamine (Gibco). Recombinant human TGF- $\beta$ (cat\# L1718; Peprotech, Rocky Hill, NJ, USA) was used at concentrations of 1,5 , or $10 \mathrm{ng} / \mathrm{mL}$. The anti-fibrotic effects of eupatilin (cat\# S3846; Selleckchem, Houston, TX, USA) were examined at final concentrations of 25,50 , and $100 \mu \mathrm{M}$. Cells were grown on a
Matrigel-coated cover glass (1:8 dilution, growth factor-reduced; Corning, Tewksbury, MA, USA) for further investigation of morphological changes and immunofluorescence (IF) analyses.

\section{Immunofluorescence and microscopy}

IF staining using an antibody against a-SMA (1:500; Abcam, Cambridge, UK) was performed as previously described [20].

\section{Quantitative real-time reverse-transcription polymerase chain reaction}

Total RNA was extracted from Ishikawa cells from three independent experiments using TRIzol reagent (Ambion, Austin, TX, USA). One microgram of RNA was used to synthesize complementary DNA using M-MLV reverse transcriptase (Promega, Madison, WI, USA) and an oligo-dT primer. With one-tenth of the volume of complementary DNA, gene expression was quantitatively analyzed using a reversetranscription polymerase chain reaction (PCR) machine (Bio-Rad, Hercules, CA, USA). Quantitative real-time PCR analysis was performed using FastStart Universal SYBR Green Master Mix (Roche, Basel, Switzerland) on a CFX Connect Real-Time PCR Detection System (Bio-Rad). Amplification consisted of 40 cycles: denaturation at $95^{\circ} \mathrm{C}$ for 10 minutes, annealing at $58^{\circ} \mathrm{C}-60^{\circ} \mathrm{C}$ for 1 minute, and extension at $72^{\circ} \mathrm{C}$ for 1 minute. The primers used in this study are listed in Table 1. The expression levels of genes of interest, which were measured by cycle threshold (Ct) values, were normalized to the $\mathrm{Ct}$ values of $\beta$-actin and evaluated as a percentage of the expression level measured in cells of the control group. More specifically, the proportional expression of the gene of interest compared to $\beta$-actin was quanti-

Table 1. Primer sequence pairs used for reverse-transcription polymerase chain reaction analyses

\begin{tabular}{ll}
\hline Gene & \multicolumn{1}{c}{ Sequence } \\
\hline $\begin{array}{l}a-\text { SMA } \\
\text { Forward } \\
\text { Reverse } \\
H I F 1 a\end{array}$ & AGCGTGGCTATTCCTTCGTT \\
Forward & TGAAGGATGGCTGGAACAGG \\
Reverse & \\
COL1A1 & CACCACAGGACAGTACAGGAT \\
Forward & CGTGCTGAATAATACCACTCAC \\
Reverse & \\
MMP-2 & AGACATCCCACCAATCACCTG \\
Forward & GGCAGTTCTTGGTCTCGTCAC \\
Reverse & \\
$\beta$ - $a c t i n$ & TGATGGCATCGCTCAGATCC \\
Forward & GGCCTCGTATACCGCATCAA \\
Reverse & \\
\hline
\end{tabular}

a-SMA, alpha-smooth muscle actin; HIF-1a, hypoxia-inducible factor 1 alpha; COL1A1, collagen type I alpha 1 chain; MMP-2, matrix metalloproteinase-2. 
fied as $2^{\mathrm{Ct}(\text { gene of interest)-Ct(B-actin) }}$.

\section{Statistical analysis}

The comparison groups were analyzed using the unpaired Student $t$ test for parametric distributions. For multiple comparisons, two-way analysis of variance was used, followed by the Sidak multiple comparison test. For all tests, a $p$-value that was $<0.05$ was considered to indicate statistical significance $(p<0.05, p<0.01, p<0.001$, and $p<0.0001)$.

\section{Results}

\section{Eupatilin restores TGF- $\beta$-induced fibrotic activity in Ishikawa cells}

The effects of eupatilin on TGF- $\beta$-induced endometrial fibrosis were investigated by assessing morphological alterations in Ishikawa cells. Prior to eupatilin application, the Ishikawa cells were pre-treated with recombinant human TGF- $\beta$ for 48 hours to induce fibrosis. As shown in Figure 1, in a dose-dependent manner more fibrotic morphology was observed with higher doses of TGF- $\beta$ (far left panel), and additional treatments with eupatilin $(25,50$, and $100 \mu \mathrm{M})$ dramatically reduced TGF- $\beta$-induced fibrotic phenotypes, as the cells displayed more circular shapes and formed more colonies, which are typical morphological features of Ishikawa cells in culture. IF staining for a-SMA, a surrogate marker for myofibroblasts, substantiated these findings of morphological alterations. IF and immunoblotting analyses demonstrated that the levels of a-SMA expression in Ishikawa cells were increased by TGF- $\beta$, and subsequent eupatilin treatment drastically inhibited TGF- $\beta$-induced $a-S M A$ to the levels detected in the control group (Figure 2). These data indicate that eupatilin acted directly to attenuate TGF- $\beta$-induced fibrotic activity and

Eupatilin treatment: 24 hours

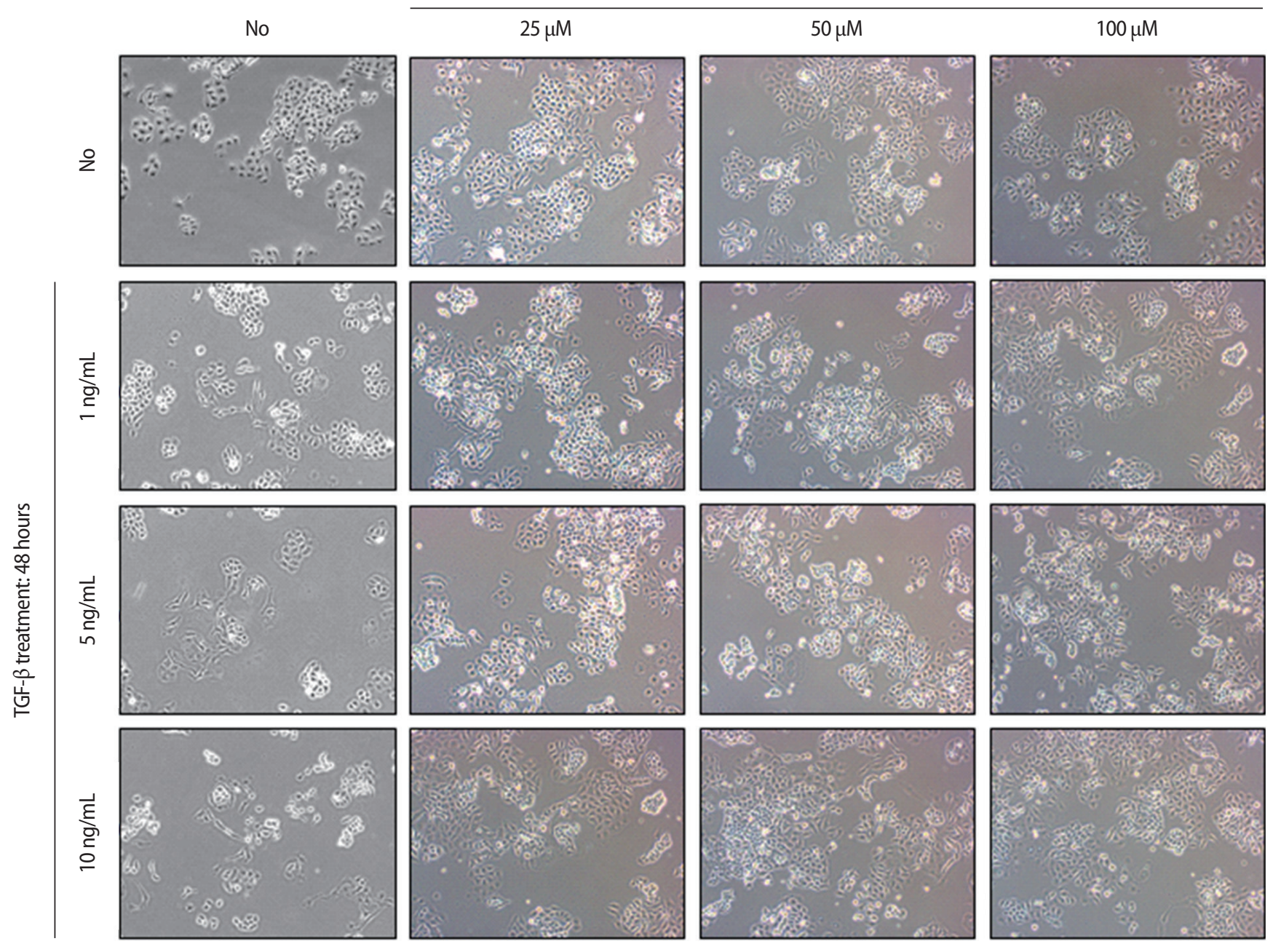

Figure 1. Eupatilin treatment inhibited transforming growth factor beta (TGF- $\beta$ )-induced fibrotic morphology in Ishikawa cells. Morphological changes in response to TGF- $\beta(1,5$, and $10 \mathrm{ng} / \mathrm{mL})$ alone, and additional treatment of eupatilin $(25,50$, and $100 \mu \mathrm{M})$. Morphological changes were monitored by phase-contrast microscopy at $\times 10$ magnification. 
restored the fibrotic features to the status of normal cells. To determine the efficacy of eupatilin in changing the gene expression of fibrosis markers, we performed quantitative PCR analyses to detect the expression of key genes involved in TGF- $\beta$-induced endometrial fibrosis in Ishikawa cells and then measured the changes in their expression levels in response to eupatilin treatment. a-SMA, hypoxia-

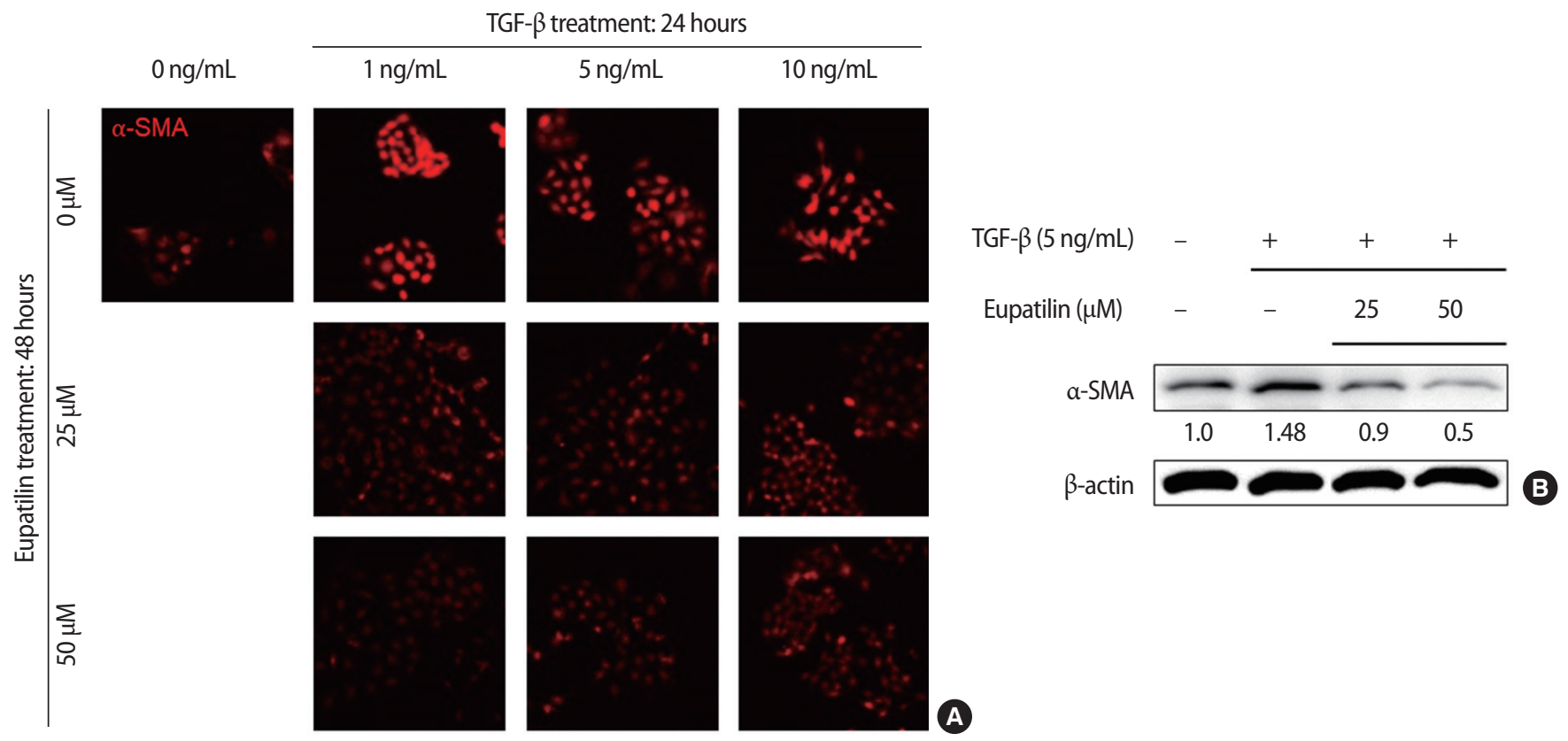

Figure 2. Eupatilin treatment restored transforming growth factor beta (TGF- $\beta$ )-induced high levels of alpha-smooth muscle actin ( $a$-SMA) expression. Changes of a-SMA expression in response to TGF- $\beta(5 \mathrm{ng} / \mathrm{mL}, 24$ hours) and additional treatment of eupatilin ( 25 or $50 \mu \mathrm{M}, 48 \mathrm{hours})$ using immunofluorescence staining (A) and immunoblotting (B) analyses. Densitometry of immunoblot bands shown in (B) was performed using Image $J$, and $\beta$-actin was used as a control.

A

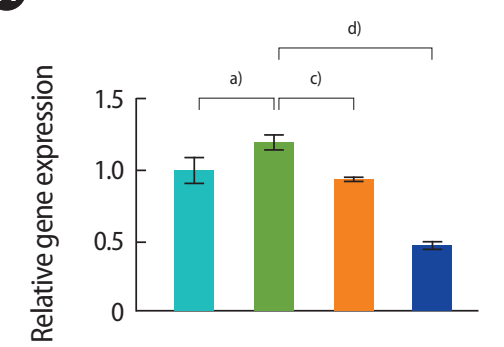

C

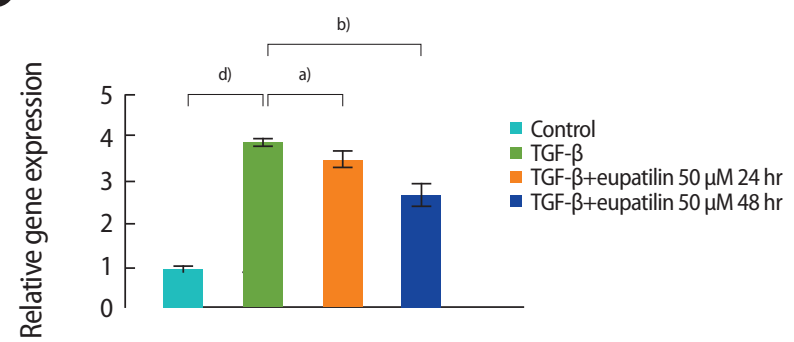

$$
\text { - Control }
$$

- TGF- $\beta$ +eupatilin $50 \mu \mathrm{M} 24 \mathrm{hr}$ - TGF- $\beta+$ eupatilin $50 \mu \mathrm{M} 48 \mathrm{hr}$

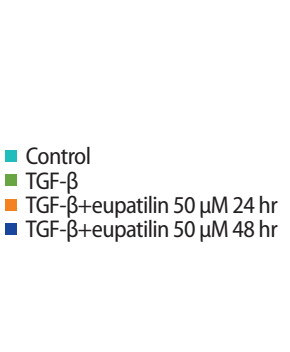

B

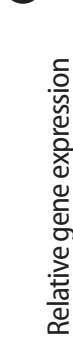

D

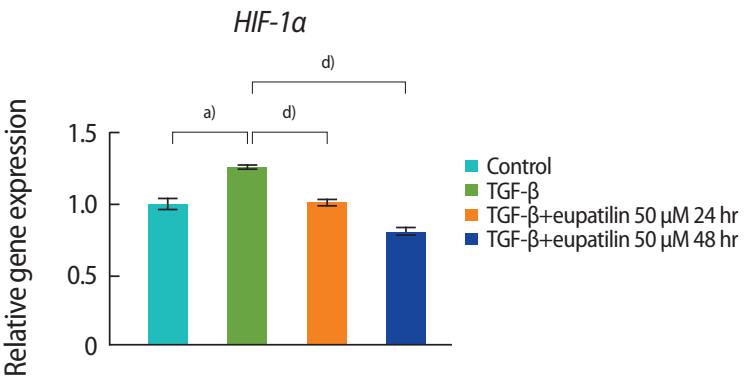

MMP-2

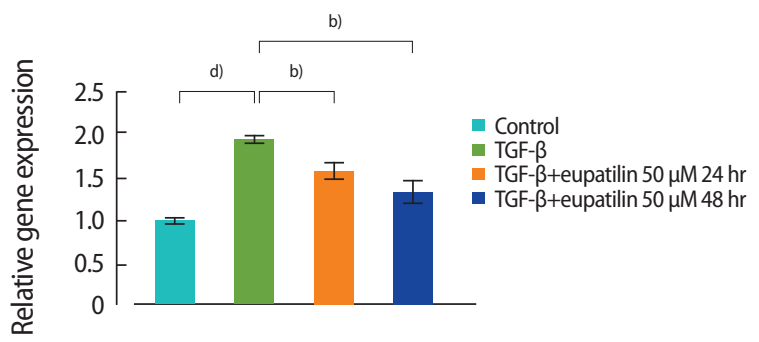

Figure 3. Quantitative real-time reverse-transcription polymerase chain reaction was used to quantify the mRNA expression of alpha-smooth muscle actin ( $\mathrm{a}-\mathrm{SMA}$; $\mathrm{A}$ ), hypoxia-inducible factor 1 alpha (HIF-1a; $\mathrm{B})$, collagen type I alpha 1 chain (COL1A1; C), and matrix metalloproteinase-2 (MMP- $2 ; \mathrm{D}$ ) in the transforming growth factor beta (TGF- $\beta$ ), TGF- $\beta+$ post-eupatilin ( $50 \mu \mathrm{M}, 24$ hours), and TGF- $\beta+$ post-eupatilin (50 $\mu \mathrm{M}, 48$ hours) groups compared to the control group. The two-tailed Student $t$-test was used to evaluate the statistical significance of differences between the experimental groups. The $p$-values were considered to indicate statistical significance as follows: ${ }^{\text {a) }} p<0.05,{ }^{\text {b) }} p<0.01,{ }^{\text {c) }} p<0.001$, and ${ }^{\text {d) }} p<0.0001$. 
inducible factor 1 alpha (HIF-1a), collagen type I alpha 1 chain (COL1A1), and matrix metalloproteinase-2 (MMP-2) were highly upregulated by 48 hours of treatment with TGF- $\beta$, and their expression was significantly reduced by treatment with $50 \mu \mathrm{M}$ eupatilin (Figure 3). Longer exposure to eupatilin resulted in a greater reduction of the expression of all four of those genes. Of note, both COL1A1 and MMP-2 failed to recover to the levels of the control cells with $25 \mu \mathrm{M}$ eupatilin treatment (data not shown), implying that certain levels of eupatilin might be required to overcome the fibrotic effects induced by TGF- $\beta$. Taken together, these data reveal that TGF- $\beta$-induced phenotypic and molecular alterations in endometrial fibrosis were both restored by eupatilin treatment, suggesting this drug as a potent therapeutic intervention for patients with fibrosis in the endometrium.

\section{Discussion}

In recent years, autologous transplantation of bone marrow-derived stem cells [21] and menstrual blood-derived stromal cells [22] has been performed in patients with endometrial fibrosis or intrauterine adhesion. Cell therapies targeting endometrial fibrosis might be an option for stimulating the growth of the endometrium and recovering reproductive function $[21,22]$. However, the molecularbased mechanisms underlying anti-fibrotic activity have not yet been fully elucidated. Previous studies have suggested that microRNAs (miRNAs) might serve as potential therapeutic targets for endometrial fibrosis. Xiao et al. [23] demonstrated that miRNA-340 induced by bone marrow mesenchymal stem cell treatment stimulated the regeneration of rat endometrial tissue subjected to mechanical damage. Furthermore, Ning et al. [2] reported that down-regulation of miRNA-326 was strongly associated with intrauterine adhesion, characterized by endometrial fibrosis and high levels of TGF- $\beta 1$, a-SMA, COL1A1, and fibronectin, as we observed in the present study. Recently, two small molecules (pirfenidone and nintedanib) were approved for idiopathic pulmonary disease, as they showed combinatorial anti-inflammatory, antioxidant, and antifibrotic effects [24,25]. Moreover, Kim et al. [18] reported that eupatilin treatment attenuated the progression of bleomycin-induced lung fibrosis and TGF- $\beta$-induced fibrotic activity in hepatic stellate cells by disturbing Smad3 phosphorylation, which subsequently led to myofibroblast dedifferentiation and reversal of fibrotic progression. They demonstrated the effects of eupatilin on actin depolymerization and suppression of induction of the epithelial-mesenchymal transition [18]. However, no report has yet provided evidence for the impact of eupatilin in endometrial fibrosis.

The present study demonstrates that eupatilin treatment restored TGF- $\beta$-induced fibrotic activity in endometrial epithelial cells, as shown by a reduction in the degree of fibrotic phenotypes and a more circular shape of individual cells. This conclusion is also supported by the significant observed reduction in the expression of a-SMA, COL1A1, and MMP- 2 , which were strongly induced by TGF- $\beta$, by eupatilin treatment. Due to technical obstacles and limitations, we evaluated the effects of eupatilin treatment on fibrosis that was artificially induced by TGF- $\beta$ in Ishikawa cells in vitro instead of using primary endometrial epithelial cells. Additionally, Ishikawa cells only represent the epithelial compartment of the endometrium. Since fibrotic activity and its progression result from communication between the endometrial epithelium and stroma, our future research will address the relevance of eupatilin treatment for fibrotic activity in endometrial stromal cells in association with the endometrial epithelial layer. In conclusion, the findings of the present study suggest that suppression of TGF- $\beta$-induced signaling by eupatilin might be an effective therapeutic strategy for the treatment of endometrial fibrosis. Preventing endometrial fibrosis by understanding its fundamental molecular mechanisms may potentially provide therapeutic strategies for converting fibrotic endometrial tissue into functionally active endometrium.

\section{Conflict of interest}

Hwa Seon Koo has been a managing editor of Journal of Clinical and Experimental Reproductive Medicine since 2018; however, she was not involved in the peer reviewer selection, evaluation, or decision process of this article. No other potential conflicts of interest relevant to this article were reported.

\section{ORCID}

Chang-Jin Lee https://orcid.org/0000-0003-4629-8052 Seon-Hwa Hong https://orcid.org/0000-0002-0663-1781 Min-Ji Yoon https://orcid.org/0000-0002-8098-7073 Kyung-Ah Lee https://orcid.org/0000-0001-6166-5012 Dong Hee Choi https://orcid.org/0000-0001-7242-8987 Jung-Jae Ko https://orcid.org/0000-0002-5361-5784 Jee Hyun Kim https://orcid.org/0000-0002-2584-108X Hwa Seon Koo https://orcid.org/0000-0003-2783-5668 Youn-Jung Kang https://orcid.org/0000-0002-0771-9515

\section{Author contributions}

Conceptualization: YJK, HSK, JJK. Data curation: CJL, SHH. Formal analysis: CJL, YJK. Funding acquisition: YJK. Methodology: MJY, KAL. Project administration: YJK, HSK, JJK. Visualization: CJL, MJY. Writingoriginal draft:YJK. Writing-review \& editing:YJK, DHC, HK. 


\section{References}

1. Ebrahim N, Mostafa O, El Dosoky RE, Ahmed IA, Saad AS, Mostafa $A$, et al. Human mesenchymal stem cell-derived extracellular vesicles/estrogen combined therapy safely ameliorates experimentally induced intrauterine adhesions in a female rat model. Stem Cell Res Ther 2018;9:175.

2. Ning J, Zhang $H$, Yang $H$. MicroRNA-326 inhibits endometrial fibrosis by regulating TGF- $\beta 1 / \mathrm{Smad} 3$ pathway in intrauterine adhesions. Mol Med Rep 2018;18:2286-92.

3. Walton $\mathrm{KL}$, Johnson $\mathrm{KE}$, Harrison $\mathrm{CA}$. Targeting TGF- $\beta$ mediated SMAD signaling for the prevention of fibrosis. Front Pharmacol 2017;8:461.

4. Karsdal MA, Manon-Jensen T, Genovese F, Kristensen JH, Nielsen MJ, Sand JM, et al. Novel insights into the function and dynamics of extracellular matrix in liver fibrosis. Am J Physiol Gastrointest Liver Physiol 2015;308:G807-30.

5. Menzies D. Postoperative adhesions: their treatment and relevance in clinical practice. Ann R Coll Surg Engl 1993;75:147-53.

6. Deans R, Abbott J. Review of intrauterine adhesions. J Minim Invasive Gynecol 2010;17:555-69.

7. Amin TN, Saridogan E, Jurkovic D. Ultrasound and intrauterine adhesions: a novel structured approach to diagnosis and management. Ultrasound Obstet Gynecol 2015;46:131-9.

8. Macneal K, Schwartz DA. The genetic and environmental causes of pulmonary fibrosis. Proc Am Thorac Soc 2012;9:120-5.

9. Pohlers D, Brenmoehl J, Loffler I, Muller CK, Leipner C, SchultzeMosgau S, et al. TGF-beta and fibrosis in different organs: molecular pathway imprints. Biochim Biophys Acta 2009;1792:746-56.

10. Nanthakumar CB, Hatley RJ, Lemma S, Gauldie J, Marshall RP, Macdonald SJ. Dissecting fibrosis: therapeutic insights from the small-molecule toolbox. Nat Rev Drug Discov 2015;14:693-720.

11. Beanes SR, Dang C, Soo C, Ting K. Skin repair and scar formation: the central role of TGF-beta. Expert Rev Mol Med 2003;5:1-22.

12. Kramer EL, Clancy JP. TGF $\beta$ as a therapeutic target in cystic fibrosis. Expert Opin Ther Targets 2018;22:177-89.

13. Tao Q, Wang XN, Mu YP, Feng Q, Peng JH, Liu P, et al. Dynamic change of lipid peroxidation-related protein expression and the intervention effects of Yiguanjian decoction in a rat model of CCl4-induced liver fibrosis. Zhonghua Gan Zang Bing Za Zhi 2012;20:116-21.
14. Willis BC, Borok Z. TGF-beta-induced EMT: mechanisms and implications for fibrotic lung disease. Am J Physiol Lung Cell Mol Physiol 2007;293:L525-34.

15. Biernacka A, Dobaczewski M, Frangogiannis NG. TGF- $\beta$ signaling in fibrosis. Growth Factors 2011;29:196-202.

16. Hinz B. Mechanical aspects of lung fibrosis: a spotlight on the myofibroblast. Proc Am Thorac Soc 2012;9:137-47.

17. Liu S, Xu SW, Kennedy L, Pala D, Chen Y, Eastwood M, et al. FAK is required for TGFbeta-induced JNK phosphorylation in fibroblasts: implications for acquisition of a matrix-remodeling phenotype. Mol Biol Cell 2007;18:2169-78.

18. Kim HS, Yoon YM, Meang MK, Park YE, Lee JY, Lee TH, et al. Reversion of in vivo fibrogenesis by novel chromone scaffolds. EBioMedicine 2019;39:484-96.

19. Nageen B, Sarfraz I, Rasul A, Hussain G, Rukhsar F, Irshad S, et al. Eupatilin: a natural pharmacologically active flavone compound with its wide range applications. J Asian Nat Prod Res 2020;22:116.

20. Kang YJ, Balter B, Csizmadia E, Haas B, Sharma H, Bronson R, et al. Contribution of classical end-joining to PTEN inactivation in p53-mediated glioblastoma formation and drug-resistant survival. Nat Commun 2017;8:14013.

21. Santamaria X, Cabanillas S, Cervello I, Arbona C, Raga F, Ferro J, et al. Autologous cell therapy with CD133+ bone marrow-derived stem cells for refractory Asherman's syndrome and endometrial atrophy: a pilot cohort study. Hum Reprod 2016;31: 1087-96.

22. Tan J, Li P, Wang Q, Li Y, Li X, Zhao D, et al. Autologous menstrual blood-derived stromal cells transplantation for severe Asherman's syndrome. Hum Reprod 2016;31:2723-9.

23. Xiao B, Zhu Y, Huang J, Wang T, Wang F, Sun S. Exosomal transfer of bone marrow mesenchymal stem cell-derived miR-340 attenuates endometrial fibrosis. Biol Open 2019;8:bio039958.

24. Rivera-Ortega P, Hayton C, Blaikley J, Leonard C, Chaudhuri N. Nintedanib in the management of idiopathic pulmonary fibrosis: clinical trial evidence and real-world experience. Ther Adv Respir Dis 2018;12:1753466618800618.

25. Taniguchi H, Ebina M, Kondoh Y, Ogura T, Azuma A, Suga M, et al. Pirfenidone in idiopathic pulmonary fibrosis. Eur Respir J 2010; 35:821-9. 